\title{
VSURF: An R Package for Variable Selection Using Random Forests
}

\author{
by Robin Genuer, Jean-Michel Poggi and Christine Tuleau-Malot
}

\begin{abstract}
This paper describes the R package VSURF. Based on random forests, and for both regression and classification problems, it returns two subsets of variables. The first is a subset of important variables including some redundancy which can be relevant for interpretation, and the second one is a smaller subset corresponding to a model trying to avoid redundancy focusing more closely on the prediction objective. The two-stage strategy is based on a preliminary ranking of the explanatory variables using the random forests permutation-based score of importance and proceeds using a stepwise forward strategy for variable introduction. The two proposals can be obtained automatically using data-driven default values, good enough to provide interesting results, but strategy can also be tuned by the user. The algorithm is illustrated on a simulated example and its applications to real datasets are presented.
\end{abstract}

\section{Introduction}

Variable selection is a crucial issue in many applied classification and regression problems (see e.g. Hastie et al., 2001). It is of interest for statistical analysis as well as for modelisation or prediction purposes to remove irrelevant variables, to select all important ones or to determine a sufficient subset for prediction. These main different objectives from a statistical learning perspective involve variable selection to simplify statistical problems, to help diagnosis and interpretation, and to speed up data processing.

Genuer et al. (2010b) proposed a variable selection method based on random forests (Breiman, 2001), and the aim of this paper is to describe the associated R package called VSURF and to illustrate its use on real datasets. The stable version of the package is available from the Comprehensive $\mathrm{R}$ Archive Network (CRAN) at http: //CRAN.R-project.org/package=VSURF. ${ }^{1}$ In order to make the paper self-contained, the description of the variable selection method is provided. A simulated toy example and a classical real dataset are processed using VSURF. In addition, two real examples in a high-dimensional setting, not previously addressed by the authors, are used to illustrate the value of the strategy and the effectiveness of the $\mathrm{R}$ package.

Introduced by Breiman (2001), random forests (abbreviated RF in the sequel) are an attractive nonparametric statistical method to deal with these problems, since they require only mild conditions on the model supposed to have generated the observed data. Indeed, since RF are based on decision trees and use aggregation ideas, they allow us to consider in an elegant and versatile framework different models and problems, namely regression, two-class and multiclass classifications.

Considering a learning set $L=\left\{\left(X_{1}, Y_{1}\right), \ldots,\left(X_{n}, Y_{n}\right)\right\}$, supposed to consist of independent observations of the random vector $(X, Y)$, we distinguish as usual the predictors (or explanatory variables), collected in the vector $X=\left(X^{1}, \ldots, X^{p}\right)$ where $X \in \mathbb{R}^{p}$, from the explained variable $Y \in \mathcal{Y}$ where $Y$ is either a class label for classification problems or a numerical response for regression ones. Let us recall that a classifier $t$ is a mapping $t: \mathbb{R}^{p} \rightarrow \mathcal{Y}$ while the regression function naturally corresponds to the function $s$ when we suppose that $Y=s(X)+\varepsilon$ with $E[\varepsilon \mid X]=0$. Then random forests provide estimators of either the Bayes classifier, which minimizes the classification error $P(Y \neq t(X))$, or the regression function.

The CART (Classification and Regression Trees) method defined by Breiman et al. (1984) is a well-known way to design optimal single binary decision trees. It proceeds by performing first a growing step and then a pruning one. The principle of random forests is to aggregate many binary decision trees coming from two random perturbation mechanisms: the use of bootstrap samples of $L$ instead of $L$ and the random choice of a subset of explanatory variables at each node instead of all of them. There are two main differences with respect to CART trees: first, in the growing step, at each node, a fixed number of input variables are randomly chosen and the best split is calculated only among them and, second, no pruning step is performed so all the trees of the forest are maximal trees. The RF algorithm is a very popular machine learning algorithm and appears to be powerful in a lot of different applications, see for example Verikas et al. (2011) and Boulesteix et al. (2012) for recent surveys.

Several implementations of these methods are available. Focusing on $\mathrm{R}$ packages we must mention rpart (Therneau et al., 2015) for CART, randomForest (Liaw and Wiener, 2002) for RF, party (Hothorn

\footnotetext{
${ }^{1}$ The current development version of the package is also available at https://gi thub. com/robingenuer/VSURF.
} 
et al., 2006) for CART and RF (through the function cforest) and ipred (Peters et al., 2002) for bagging (Breiman, 1996), a closely related method cited here for the sake of completeness. In this paper, we use the randomForest procedure, which is based on the initial contribution of Breiman and Cutler (2004). We will concentrate on the prediction performance of RF focusing on the out-of-bag (OOB) error (see Breiman, 2001) and on the quantification of the variable importance (VI in the sequel) which are key ingredients for our variable selection strategy. For a general discussion about variable importance, see Azen and Budescu (2003). In the random forests framework, one of the most widely used scores of importance of a given variable is the increase in mean of the error of a tree (mean square error (MSE) for regression and misclassification rate for classification) in the forest when the observed values of this variable are randomly permuted in the OOB samples (see Archer and Kimes, 2008).

Strobl et al. (2007) showed that VI scores are biased towards correlated variables, and Strobl et al. (2008) proposed an alternative permutation scheme as a solution, which, however, increases the computation cost. This seems to be especially critical for high-dimensional problems with strongly correlated predictors. Nevertheless, our previous experiments (Genuer et al., 2010b) on variable selection and, more recently, the theoretical study of Gregorutti et al. (2013) show that, in some situations, the VI scores are biased towards uncorrelated variables. Additional theoretical results and experiments are needed to more deeply understand these phenomenons, but this is out of the scope of the paper.

A lot of variable selection procedures are based on the combination of variable importance for ranking and model estimation to generate, evaluate and compare a family of models, i.e., in particular in the family of "wrapper" methods (Kohavi and John, 1997; Guyon and Elisseeff, 2003) which include the prediction performance in the score calculation, for which a lot of methods can be cited. We choose to highlight one of them which is widely used and close to our procedure. Díaz-Uriarte and Alvarez De Andres (2006) propose a strategy based on recursive elimination of variables. At the beginning, they compute RF variable importance and then, at each step, eliminate iteratively the $20 \%$ of the variables having the smallest importance and build a new forest with the remaining variables. The final set of variables is selected by minimizing over the obtained forests, the OOB error rate defined by:

$$
\operatorname{err} O O B= \begin{cases}\frac{1}{n} \operatorname{Card}\left\{i \in\{1, \ldots, n\} \mid y_{i} \neq \hat{y}_{i}\right\} & \text { in the classification framework } \\ \frac{1}{n} \sum_{i \in\{1, \ldots, n\}}\left(y_{i}-\hat{y}_{i}\right)^{2} & \text { in the regression framework }\end{cases}
$$

where $\hat{y}_{i}$ is the aggregation of the predicted values by trees $t$ for which $\left(x_{i}, y_{i}\right)$ belongs to the associated $O O B$ sample (data not included in the bootstrap sample used to construct $t$ ). The proportion of variables to eliminate is an arbitrary parameter of their method and does not depend on the data. Let us remark that we propose an heuristic strategy which does not depend on specific model hypotheses, but which is based on data-driven thresholds to take decisions.

This topic of variable selection still continues to be of interest. Indeed recently Hapfelmeier and Ulm (2012) propose a new variable selection approach using random forests and, more generally, Cadenas et al. (2013) describe and compare different approaches in a survey paper.

Some packages are available to cope with variable selection problems. Let us cite, for classification problems the R package Boruta, described in Kursa and Rudnicki (2010), which aims at finding all relevant variables using a random forest classification algorithm which iteratively removes the variables using a statistical test. The $R$ package varSelRF, described in Díaz-Uriarte (2007), implements the previously described method for selecting very small sets of genes in the context of classification. The R package ofw (Lê Cao and Chabrier, 2008), also dedicated to the context of classification, selects relevant variables based on the application of supervised multiclass classifiers such as CART or support vector machines. The R package spikeSlabGAM implements Bayesian variable selection via regularized estimation in additive mixed models (Scheipl, 2011). Dedicated to the biomarker identification in the life sciences, the R package BioMark implements two meta-statistics for variable selection (Wehrens et al., 2012): the first sets a data-dependent selection threshold for significance, which is useful when two groups are compared, and the second, more general one, uses repeated subsampling and selects the model coefficients remaining consistently important.

The paper is organized as follows. After this introduction, we present the general variable selection strategy. We then describe how to use package VSURF on a simple simulated dataset. Finally, we examine three real-life examples to illustrate the method in action in high-dimensional situations as well as in a standard one. 


\section{The strategy}

\section{Objectives}

In Genuer et al. (2010b) we distinguished two variable selection objectives referred to as interpretation and prediction.

Even if this distinction can be a little bit confusing since we use for both objectives the same criterion related to prediction performance, the idea is the following. The first objective, called interpretation, is to find important variables highly related to the response variable, even with some redundancy, possibly high. The second one, namely prediction, is to find a smaller number of variables with very low redundancy and sufficient for a good enough prediction of the response variable. The terminology used here can be misunderstood since usually for interpretation, one usually looks for parsimony but in many situations one may often want to identify all predictor variables associated with the response to interpret correctly the relation. We thank one anonymous reviewer for raising this issue and we detail an example in the next paragraph to clarify the difference.

A typical situation illustrates the distinction between the two kinds of variable selection. Let us consider a high-dimensional $(n \ll p)$ classification problem for which the predictor variables are associated to a pixel in an image or a voxel in a 3D-image as in fMRI brain activity classification problems (see e.g. Genuer et al., 2010a). In such situations, it is supposed that a lot of variables are useless and that there exist unknown groups of highly correlated predictors corresponding to brain regions involved in the response to a given stimulation. The two distinct objectives about variable selection can be of interest. Finding all the important variables highly related to the response variable is useful for interpretation, since it corresponds to the determination of entire regions in the brain or a full parcel in an image. By contrast, finding a small number of variables sufficient for good prediction allows to get the most discriminant variables within the previously highlighted regions. For a more formal approach to this distinction, see also the interesting paper Nilsson et al. (2007).

\section{Principle}

The principle of the two-steps algorithm is the following. First, we rank the variables according to a variable importance measure and the unimportant ones are eliminated. Second, we provide two different subsets obtained either by considering a collection of nested RF models and selecting the variables of the most accurate one, or by introducing sequentially the sorted variables.

Since the quantification of the variable importance is crucial for our procedure, let us recall the definition of RF variable importance. For each tree $t$ of the forest, consider the associated $O O B_{t}$ sample (data not included in the bootstrap sample used to construct $t$ ). Denote by errOOB $t$ the error (MSE for regression and misclassification rate for classification) of a single tree $t$ on this $O O B_{t}$ sample. Now, randomly permute the values of $X^{j}$ in $O O B_{t}$ to get a perturbed sample denoted by $\widetilde{O O B}_{t}^{j}$ and compute err $\widetilde{O O B_{t}}{ }^{j}$, the error of predictor $t$ on the perturbed sample. Variable importance of $X^{j}$ is then equal to:

$$
V I\left(X^{j}\right)=\frac{1}{n \text { tree }} \sum_{t}\left(\text { erroOB } \widetilde{O}_{t}^{j}-\text { errOOB }_{t}\right),
$$

where the sum is over all trees $t$ of the RF and ntree denotes the number of trees of the RF. Notice that we use this definition of importance and not the normalized one. Indeed, instead of considering (as mentioned in Breiman and Cutler, 2004) that the raw VI are independent replicates, normalizing them and assuming normality of these scores, we prefer a fully data-driven solution. This is a key point of our strategy: we prefer to estimate directly the variability of importance across repetitions of forests instead of using normality when ntree is sufficiently large, which is only valid under some specific conditions. Those conditions are difficult to check since their validity depends heavily on tuning parameters and problem peculiarities, so data-driven normalization prevents some misspecified asymptotic behavior.

Another useful argument, which provides the rationale for this kind of variable selection procedure based on a classical stepwise method combined with the use of a VI measure, is that a variable not included in the underlying true model has a null "theoretical" importance. In a recent paper Gregorutti et al. (2013) theoretically state that, in the case of additive models, irrelevant variables have null "theoretical" VI. A similar result for the mean decrease impurity index (not used in this paper) has been proven by Louppe et al. (2013). 


\section{Detailed strategy}

Let us now describe more precisely our two-steps procedure. Note that each RF is typically built using ntree $=2000$ trees.

- Step 1. Preliminary elimination and ranking:

- Rank the variables by sorting the VI (averaged over typically 50 RF runs) in descending order.

- Eliminate the variables of small importance (let $m$ denote the number of remaining variables).

More precisely, starting from this order, consider the ordered sequence of the corresponding standard deviations (sd) of VI and use it to estimate a threshold value for VI. Since variability of VI is larger for true variables compared to useless ones, the threshold value is given by an estimation of the VI standard deviation of the useless variables. This threshold is set to the minimum prediction value given by a CART model where the $Y$ are the sd of the VI and the $X$ are the ranks.

Then only the variables with an averaged VI exceeding this threshold are retained.

- Step 2. Variable selection:

- For interpretation: construct the nested collection of RF models involving the $k$ first variables, for $k=1$ to $m$ and select the variables involved in the model leading to the smallest OOB error. This leads to consider $m^{\prime}$ variables.

More precisely, we compute OOB error rates of RF (averaged typically over 25 runs) of the nested models starting from the one with only the most important variable, and ending with the one including all important variables previously kept. Ideally, the variables of the model leading to the smallest OOB error are selected. In fact, in order to deal with instability, we use a classical trick: we select the smallest model with an OOB error less than the minimal OOB error augmented by its standard deviation (based on the same 25 runs).

- For prediction: starting with the ordered variables retained for interpretation, construct an ascending sequence of RF models, by invoking and testing the variables in a stepwise way. The variables of the last model are selected.

More precisely, the sequential variable introduction is based on the following test: a variable is added only if the error decrease is larger than a threshold. The idea is that the OOB error decrease must be significantly greater than the average variation obtained by adding noisy variables. The threshold is set to the mean of the absolute values of the first order differentiated OOB errors between the model with $m^{\prime}$ variables and the one with $m$ variables:

$$
\frac{1}{m-m^{\prime}} \sum_{j=m^{\prime}}^{m-1}|\operatorname{errOOB}(j+1)-\operatorname{errOOB}(j),|
$$

where $\operatorname{errOOB}(j)$ is the OOB error of the RF built using the $j$ most important variables.

\section{Comments}

In addition to the detailed strategy, let us give some additional comments. Regarding the first step of our procedure, our previous simulation study in Genuer et al. (2010b) shows that both VI level and variability are larger for relevant variables.

We assume that the stabilized ranking performed in the first step of our procedure will not change after the elimination step.

The use of CART to find the threshold is of course not strictly necessary and can be replaced by many other strategies but it is interesting in our case because the idea is typically to find a constant on a large interval. Since CART fits a piece-wise constant function, this desired constant is the threshold for VI and is defined as the minimum prediction value given by a CART model fitting the curve of VI standard deviations. It is obtained automatically by the CART strategy whereas the user needs to select some window parameter if, for example, a simple smoothing method is used.

Note that the threshold value is based on VI standard deviations while the effective thresholding is performed on the VI mean. In general, this rule is conservative and leads to keep more variables than necessary, postponing to the next step a more parsimonious choice.

In addition, we note that several implementation choices have been made: randomForest for RF fitting and VI calculation (repeated 50 times to quantify variability) and rpart for estimating the VI 
variability level associated with irrelevant variables leading to a convenient threshold value. Of course other choices would be possible, for example, using the function cforest of the package party to implement the same scheme of variable selection.

The next section describes the use of the package VSURF together with a complete illustration of the strategy which is not supported by specific model hypotheses but based on data-driven thresholds to take decisions.

But before entering in this description, let us emphasize that the objects of interest here are the subsets of important variables and not precisely the corresponding models. Thus OOB errors from the figures (or from the fitted objects) cannot be used or reported as the prediction error estimation of the final model because this would need a proper cross-validation scheme.

\section{Using the package VSURF}

\section{From data to final results}

We illustrate the use of the VSURF package on a simple simulated moderately high-dimensional dataset $(n=100<p=200$ ) called toys data, introduced by Weston et al. (2003). It comes from an equiprobable two-class problem, $Y \in\{-1,1\}$, with 6 influential variables and with the others being noise variables. Let us define the simulation model by giving the conditional distribution of the $X^{i}$ for $Y=y$ :

- For the six first variables: with probability $0.7, X^{i} \sim \mathcal{N}(y i, 1)$ for $i=1,2,3$ and $X^{i} \sim \mathcal{N}(0,1)$ for $i=4,5,6$; with probability $0.3, X^{i} \sim \mathcal{N}(0,1)$ for $i=1,2,3$ and $X^{i} \sim \mathcal{N}(y(i-3), 1)$ for $i=4,5,6$.

- The remaining variables are noise: $X^{i} \sim \mathcal{N}(0,1)$ for $i=7, \ldots, p$.

The variables obtained in the simulation are standardized before further analysis.

First, we load the VSURF package and the toys data (and fix the random number generation seed for reproducibility):

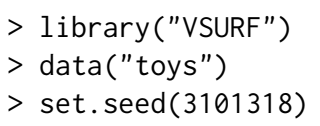

The standard way to use the VSURF package is to use the VSURF function. This function executes the complete procedure, and is just a wrapper for the three intermediate functions VSURF_thres, VSURF_interp and VSURF_pred which are described in the next section. Typical use of the VSURF function is as follows:

$>$ toys.vsurf <- $\operatorname{VSURF}(x=$ toys $\$ x, y=$ toys $\$ y, \operatorname{mtry}=100)$

The only mandatory inputs are $x$, an object containing input variables, and $y$, the output variable.

In this example, we also choose a specific value for mtry (default is $p / 3$ for classification and regression problems in VSURF), which only affects RF runs in the first step of the procedure. In addition, we stress that the default value for ntree is 2000 in VSURF. Those values were considered as well adapted for VI calculations (see Genuer et al., 2010b, Section 2.2) and these two arguments are passed to the randomForest function (we kept the same name for consistency).

The function outputs a list containing all results.

$>$ names(toys.vsurf)
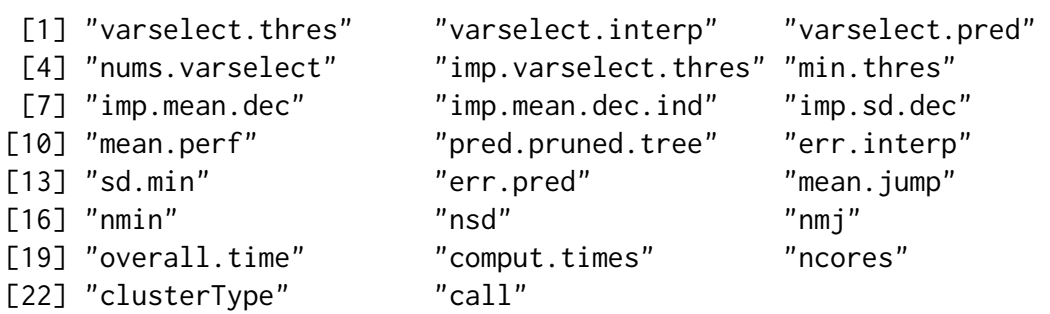

The most important objects are varselect. thres, varselect. interp and varselect. pred, which contain the set of variables selected after the thresholding, interpretation and prediction step respectively.

The summary method gives a short summary of the results: numbers of selected variables and computation times. 

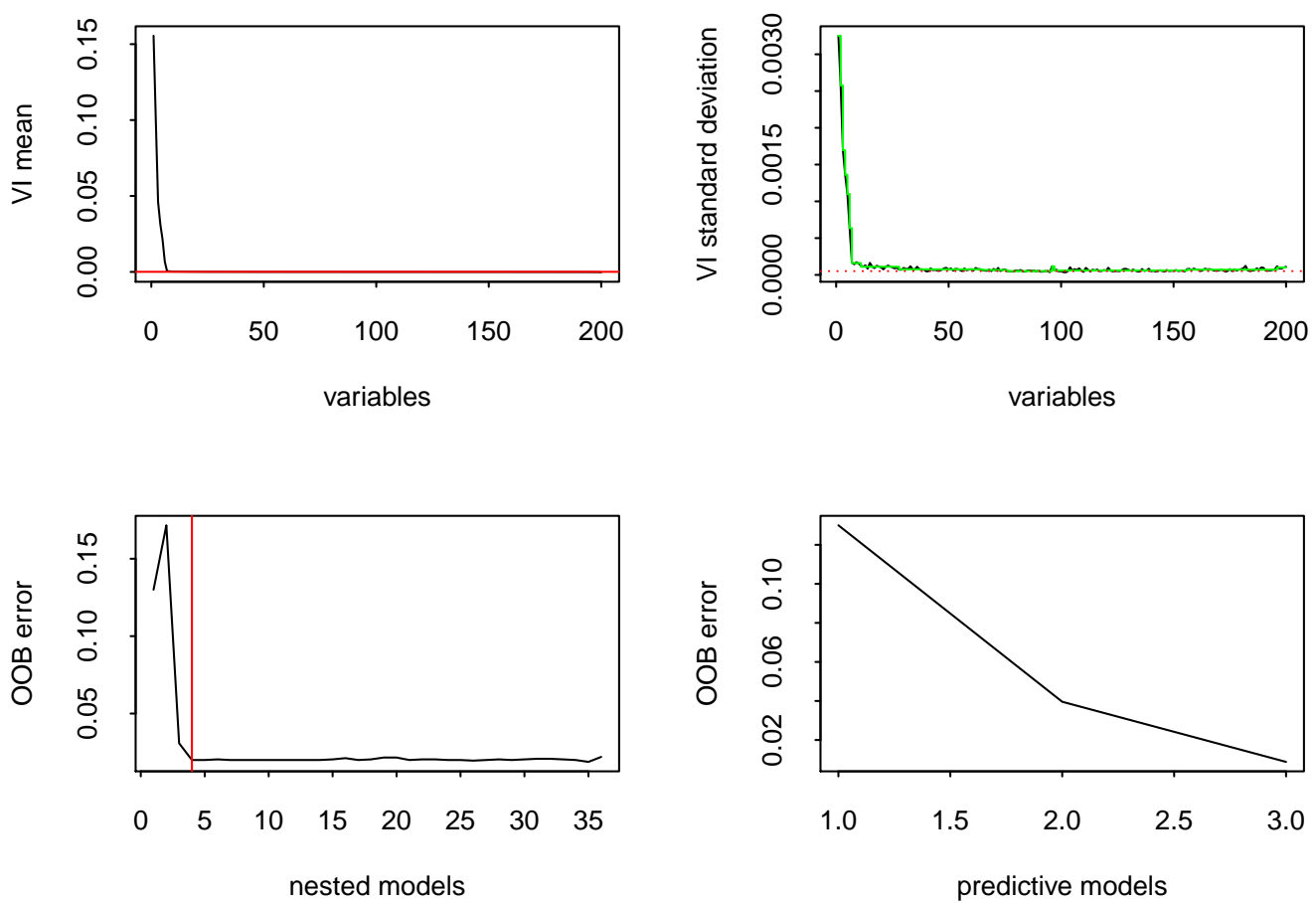

Figure 1: VSURF for the toys data: Top graphs illustrate the thresholding step, bottom left and bottom right graphs are associated with interpretation and prediction steps respectively.

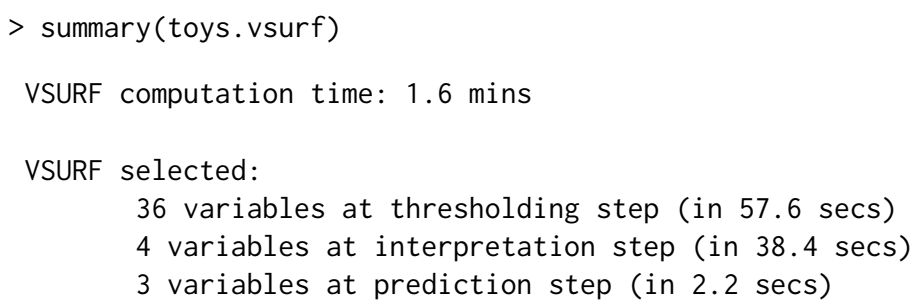

The plot method gives a plot (see Figure 1) of the results in 4 graphs. To selectively obtain single graphs of Figure 1 one can either suitably specify the arguments of this plot method, or use intermediate plot methods (associated with each procedure step) included in the package.

\section{$>$ plot(toys.vsurf)}

In addition, we include a predict method, which permits to predict the outcomes for new data with RF using only the variables selected by VSURF.

Finally, we point out that all computations of the package can be executed in parallel, whenever it is possible. For example, the following command runs VSURF in parallel on a Linux computing server using 40 cores:

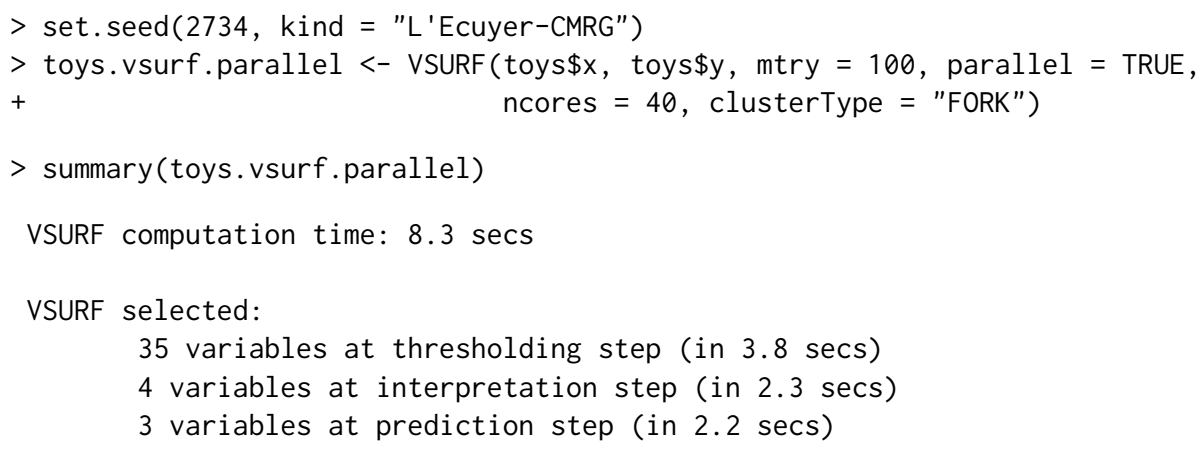

VSURF ran in parallel on a FORK cluster and used 40 cores

Note that we use the "L'Ecuyer-CMRG" kind in the set.seed function to allow reproducibility (when the call is on a FORK cluster with the same number of cores). Even if one should have a 


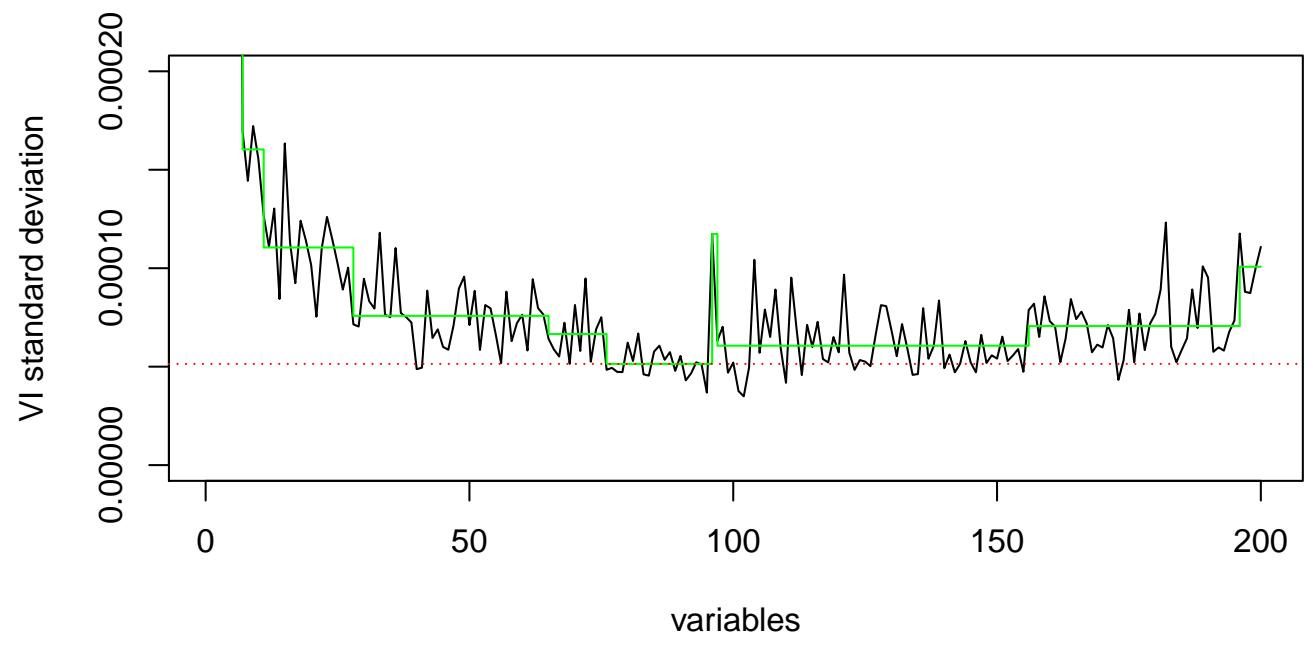

Figure 2: Zoom of the top right graph of Figure 1.

computing server with 40 cores to benefit from this execution time reduction, the resulting 'VSURF' object from this call will be the same on a computer with fewer cores available. Parallel calls of VSURF will be used to deal with the two high-dimensional datasets at the end of the paper.

\section{How to get intermediate results}

Let us now detail the main stages of the procedure together with the results obtained on the toys data. Note that unless explicitly stated otherwise, all graphs refer to Figure 1.

- Step 1.

- Variable ranking. The result of variable ranking is drawn on the top left graph. True variables are significantly more important than the noisy ones.

- Variable elimination.

Starting from this order, the plot of the corresponding standard deviations of VI is used to estimate a threshold value for VI. This threshold (figured by the dotted horizontal red line in Figure 2, which is a zoom of the top right graph of Figure 1) is set to the minimum prediction value given by a CART model fitting this curve (see the green piece-wise constant function on the same graph).

Then only the variables with an averaged VI exceeding this level (i.e. above the horizontal red line in the top left graph of Figures 1) are retained.

The computation of the 50 forests, the ranking and elimination steps are obtained with the VSURF_thres function:

$>$ set. $\operatorname{seed}(3101318)$

$>$ toys.thres <- VSURF_thres (toys $\$ x$, toys $\$ y$, mtry $=100$ )

The VSURF_thres function outputs a list containing all results of this step. The main outputs are: varselect. thres which contains the indices of variables selected by this step, imp. mean. dec and imp.sd. dec which hold the VI mean and standard deviation (the order according to decreasing VI mean can be found in imp. mean. dec.ind).

$>$ toys.thres\$varselect.thres

$\begin{array}{rrrrrrrrrrrrrrrrr}{[1]} & 3 & 2 & 6 & 5 & 1 & 4 & 184 & 37 & 138 & 159 & 81 & 17 & 180 & 131 & 52 & 191 \\ {[17]} & 96 & 192 & 165 & 94 & 198 & 25 & 21 & 109 & 64 & 12 & 29 & 188 & 107 & 157 & 70 & 46 \\ {[33]} & 54 & 143 & 186 & 111 & & & & & & & & & & & & \end{array}$

Finally, Figure 2 can be obtained with the following command:

$>$ plot(toys.vsurf, step $=$ "thres", imp.mean $=$ FALSE, ylim $=c(0,2 e-4)$ )

We can see in the plot of the VI standard deviations (top right graph of Figure 1) that the true variables' standard deviations are large compared to those of the noisy variables, which are close to zero. 
- Step 2.

- Variable selection procedure for interpretation.

We use VSURF_interp for this step. Note that we have to specify the indices of variables selected at the previous step. So we set argument vars to toys. thres $\$$ varselect. thres:

$>$ toys.interp <- VSURF_interp(toys $\$ \mathrm{x}$, toys $\$ y$,

$+\quad$ vars $=$ toys. thres $\$$ varselect.thres)

The list resulting from the VSURF_interp function mainly contains varselect. interp: the variables selected by this step, and err. interp: OOB error rates of RF nested models.

$>$ toys.interp\$varselect.interp

[1] $3 \begin{array}{llll}3 & 6 & 5\end{array}$

In the bottom left graph, we see that the error decreases quickly. It reaches its (almost) minimum when the first four true variables are included in the model (see the vertical red line) and then it remains nearly constant. The selected model contains variables V3, V2, V6, V5, which are four of the six true variables, while the actual minimum is reached for 35 variables.

Note that, to ensure quality of OOB error estimations (e.g. Genuer et al., 2008, Section 2) along embedded RF models, the mtry parameter of the randomforest function is here set to its default value if $k$ (the number of variables involved in the current RF model) is not greater than $n$, while it is set to $k / 3$ otherwise.

- Variable selection procedure for prediction.

We use the VSURF_pred function for this step. We need to specify the error rates and the variables selected in the interpretation step in the err. interp and varselect. interp arguments:

$>$ toys.pred <- VSURF_pred(toys $\$ x$, toys $\$ y$,

$+\quad$ err.interp = toys. interp\$err.interp,

$+\quad$ varselect.interp = toys.interp\$varselect.interp)

The main outputs of the VSURF_pred function are the variables selected by this final step, varselect.pred, and the OOB error rates of RF models, err. pred.

$>$ toys.pred\$varselect.pred

[1] 365

For the toys data, the final model for prediction purpose involves only variables V3, V6, V5 (see the bottom right graph). The threshold is set to the mean of the absolute values of the first order differentiated OOB errors between the model with $m^{\prime}=4$ variables and the one with $m=36$ variables.

Finally, we mention that VSURF_thres and VSURF_interp can be executed in parallel with the same syntax as VSURF (setting parallel = TRUE), while VSURF_pred cannot be parallelized.

\section{Tuning the different steps of the procedure}

We provide two additional functions for tuning the thresholding and interpretation steps without having to rerun all computations.

- First, a tune method which, applied to the result of VSURF_thres, can be used to tune the thresholding step. We can use the nmin parameter (which has default value 1) in order to set the threshold to the minimum prediction value given by the CART model times nmin.

$>$ toys.thres.tuned $<-$ tune (toys.thres, nmin $=3$ )

$>$ toys. thres. tuned\$varselect. thres

[1] $\begin{array}{llllllllllllllll}3 & 2 & 6 & 5 & 1 & 4 & 184 & 37 & 138 & 159 & 81 & 17 & 180 & 131 & 52 & 191\end{array}$

We get 16 selected variables instead of 36 previously.

- Secondly, a tune method which, applied to the result of VSURF_interp, is of the same kind and allows to tune the interpretation step. If we now want to be more restrictive in our selection in the interpretation step, we can select the smallest model with an OOB error less than the minimal OOB error augmented by its empirical standard deviation times $n s d$ (with $n s d \geq 1$ ).

$>$ toys.interp.tuned $<-$ tune(toys.interp, nsd $=5$ )

$>$ toys.interp. tuned\$varselect.interp 
[1] 326

We get 3 selected variables instead of 4 previously.

We did not write a tuning method for the prediction step because it is a recursive step and needs to recompute the sequence. Hence, to adjust the parameter for this step, we have to rerun the VSURF_pred function with a different value of $\mathrm{nmj}$ (which has default value 1). This multiplicative constant allows to modulate the threshold defined in (1).

For example, increasing the value of $n m j$ leads to selection of fewer variables:

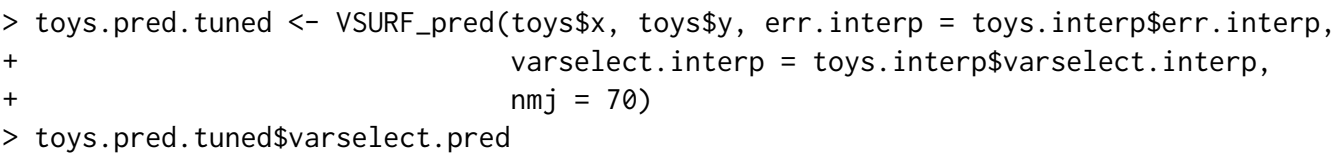

[1] 36

\section{Remark}

Thanks to one of the three anonymous reviewers, we would like to give a warning following a remark made by Svetnik et al. (2004). Indeed, this work considers a situation where there is no link between $X$ and $Y$ and for which they use OOB errors in a recursive strategy, different but not too far from our procedure, to select variables. Their results show that this kind of strategy can be seriously biased and can overfit the data. To illustrate explicitly this phenomenon, let us start with the original dataset toys for which our procedure performs quite well (see the paper Genuer et al., 2010b, containing an extensive study of the operating characteristics of the algorithm including no overfitting in presence of many additional dummy variables). Then, modify it by scrambling the $Y$ values thus removing the link between $X$ and $Y$. Applying VSURF on this modified dataset leads to an OOB error rate, in the interpretation step, starting from $50 \%$ (which is correct) and exhibiting a minimum for 10 variables corresponding to $37 \%$. So, in this situation for which there is no optimal solution and the desirable behavior is to find a constant OOB error rate along the sequence, the procedure still provides a solution. So, the conclusion is that even when there is no link between $X$ and $Y$ the procedure can highlight a set of variables.

Of course, using an external 5-fold cross-validation of our entire procedure leads to the correct estimate of $51 \%$ error rate for both interpretation and prediction steps and the correct conclusion: there is nothing to find in the data. Alternatively, if we simulate a second sample coming from the same simulation model and use it only to rank the variables, the interpretation step exhibits an OOB error rate curve oscillating around $50 \%$, which leads to the correct conclusion.

\section{Three illustrative examples}

In this section we apply the proposed procedure on three real-life examples: two high-dimensional datasets (associated with a regression problem and a classification one respectively) and, before that, a standard one to illustrate the versatility of the procedure.

Let us mention that the VSURF stability is a natural issue to investigate (see e.g. Meinshausen and Bühlmann, 2010) and is considered in Genuer et al. (2010b) Sections 3 and 4.

\section{Ozone data}

The Ozone dataset consists of $n=366$ observations for 12 independent variables and 1 dependent variable. These variables are numbered as in the R package mlbench (Leisch and Dimitriadou, 2010): 1 - Month, 2 - Day of month, 3 - Day of week, 5 - Pressure height, 6 - Wind speed, 7 - Humidity, 8 - Temperature (Sandburg), 9 - Temperature (El Monte), 10 - Inversion base height, 11 - Pressure gradient, 12 - Inversion base temperature, 13 - Visibility, for independent variables and 4 - Daily maximum one-hour-average ozone, for the dependent variable.

What makes the use of this dataset interesting, is that it has already been extensively studied and that even though it is a real one, it is possible to a priori know which variables are expected to be important. Moreover, this dataset, which is not a high-dimensional one, includes some missing data, allowing us to give an example of how to handle such data using VSURF.

To begin, we load the data:

$>\operatorname{data}("$ Ozone", package $=$ "mlbench")

$>$ set.seed(221921186) 


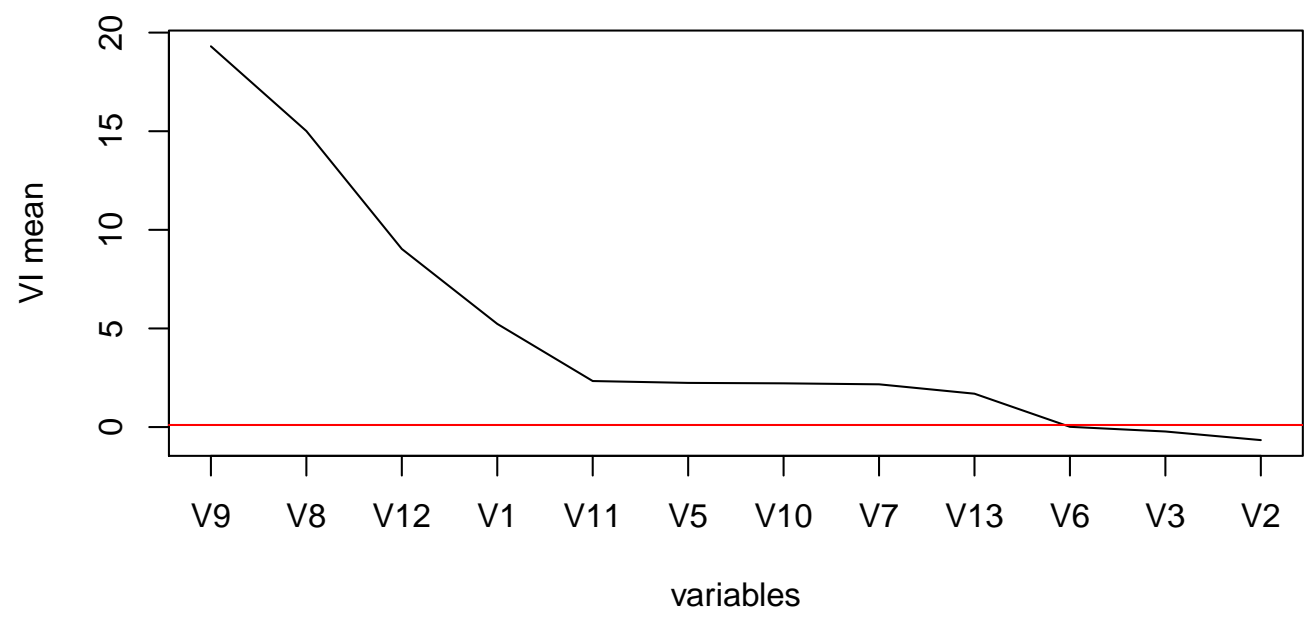

Figure 3: Sorted VI mean associated with the 12 explanatory variables of the Ozone data, with variable names on the $x$-axis.

Then, we apply the complete procedure via VSURF. Note that the following formula-type call is necessary to handle missing values (as in randomforest).

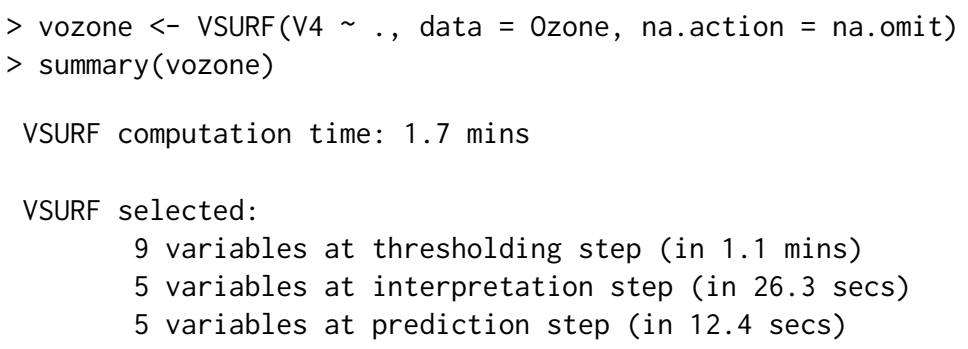

In the first step, we look at the variable importance associated with each of the explanatory variables.

$>\operatorname{plot}($ vozone, step $=$ "thres", imp.sd = FALSE, var. names $=$ TRUE)

In Figure 3, and as noticed in previous studies, three very sensible groups of variables can be discerned ranging from the most to the least important. The first group contains the two temperatures (8 and 9), the inversion base temperature (12) known to be the best ozone predictors, and the month (1), which is an important predictor since ozone concentration exhibits an heavy seasonal component. The second group of clearly less important meteorological variables consists of: pressure height (5), humidity (7), inversion base height (10), pressure gradient (11) and visibility (13). Finally the last group contains three unimportant variables: day of month (2), day of week (3) of course and more surprisingly wind speed (6). This last fact is classical: wind enters in the model only when ozone pollution arises, otherwise wind and pollution are weakly correlated (see for example Chèze et al., 2003, who highlight this phenomenon using partial estimators).

Let us now examine the results of the selection procedures. To reflect the order used in the definition of the variables, we reorder the output variables of the procedure.

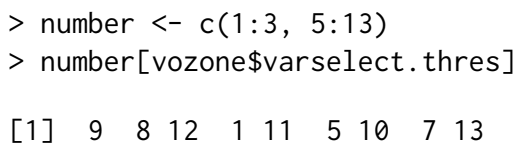

After the first elimination step, the 3 variables of negative importance (variables 6, 3 and 2) are eliminated, as expected.

$>$ number [vozone\$varselect.interp]

[1] $\begin{array}{llllll}9 & 8 & 12 & 1 & 11\end{array}$

Then the interpretation procedure leads to select the model with 5 variables, which contains all of the most important variables. 
$>$ number [vozone\$varselect.pred]

[1] $\quad \begin{array}{lllll}9 & 8 & 12 & 1 & 11\end{array}$

With the default settings, the prediction step does not remove any additional variable.

Remark

Even though the comparison with other variable selection strategies is out of the scope of the paper, one of the three anonymous reviewers has kindly compared the results of the VSURF package with the results of the R package Boruta, described in Kursa and Rudnicki (2010), on the two datasets toys and Ozone.

Let us recall that this module directly aims at selecting all-relevant features. Hence the comparison with the interpretation set delivered by VSURF is of interest. In the toys dataset, the number of truly relevant variables is 6 . VSURF finds 4 out of 6 variables in the interpretation stage while Boruta finds all six truly relevant variables and one more false positive variable. In the case of the Ozone data set VSURF finds 5 variables in the interpretation stage, while Boruta finds 9. So, this seems to confirm that the heuristic proposed by VSURF is prediction oriented (the price to pay is a risk of false negatives) and suggests that the strategy proposed by Boruta is more accurate to recover weak redundant correlations between predictors and decision variable (the price to pay seems to be a risk of false positives).

In fact our strategy assumes more or less that there are unnecessary variables in the set of all available variables initially, which is not really the case in the Ozone dataset. However, it is the case in the following two high-dimensional examples.

\section{Toxicity data}

This second dataset is also a regression framework, however, unlike before, this case is a highdimensional problem. The liver . toxicity dataset, available in the R package mixOmics (Lê Cao et al., 2015), is a real dataset from a study by Heinloth et al. (2004). In this study, 4 male rats of the inbred strain Fisher 344 were exposed to different doses of acetaminophen (non toxic dose (50 or $150 \mathrm{mg} / \mathrm{kg})$, moderate toxic dose $(1500 \mathrm{mg} / \mathrm{kg})$, severe toxic dose $(2000 \mathrm{mg} / \mathrm{kg}))$ in a controlled experiment. Necropsies were performed at different hours after exposure $(6,18,24$ and 48 hours) and the mRNA from the liver was extracted. In the original study, 10 clinical chemistry variables containing markers for the liver injury were measured. Those variables are numerical variables since they measure the serum enzymes level. For our analysis, the dataset extracted from this study contains:

- a data frame, called gene, with 64 rows representing the subjects and 3116 columns representing explanatory variables which are the gene expression levels after normalization and preprocessing due to Bushel et al. (2007),

- a vector, called clinic, with 64 rows and 1 column, one of the 10 clinical variables for the same 64 subjects: more precisely, the variable named ALB.g.dL., which corresponds to the albumin level and which is the one considered in González et al. (2012).

As in previous studies (Gidskehaug et al., 2007; Lê Cao et al., 2008), our aim is, using VSURF, to predict our clinical variable by the genes.

First, we load the data as follows:

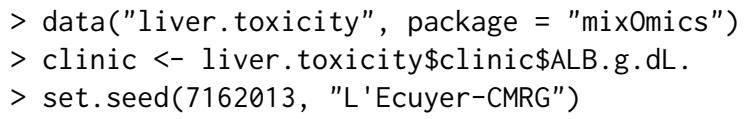

Now we apply our procedure and analyze the results.

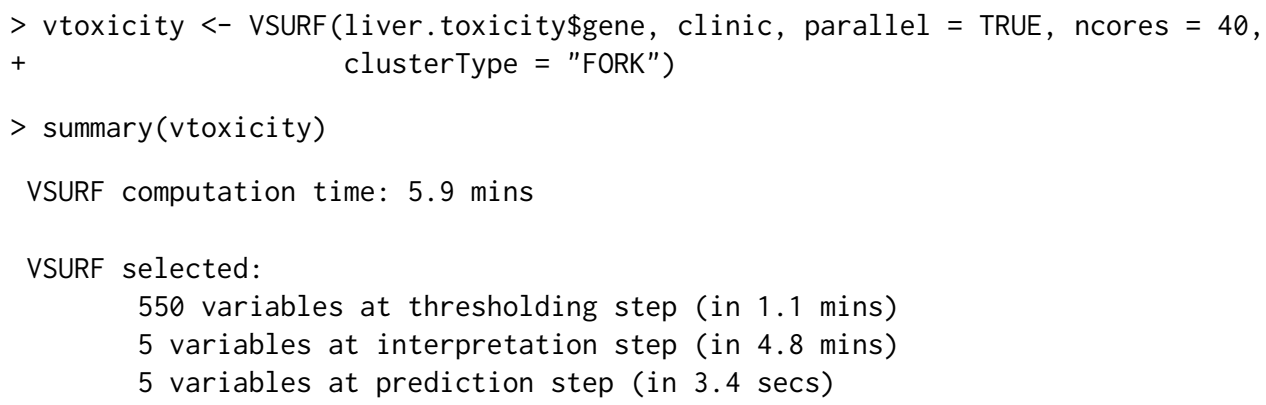

VSURF ran in parallel on a FORK cluster and used 40 cores 

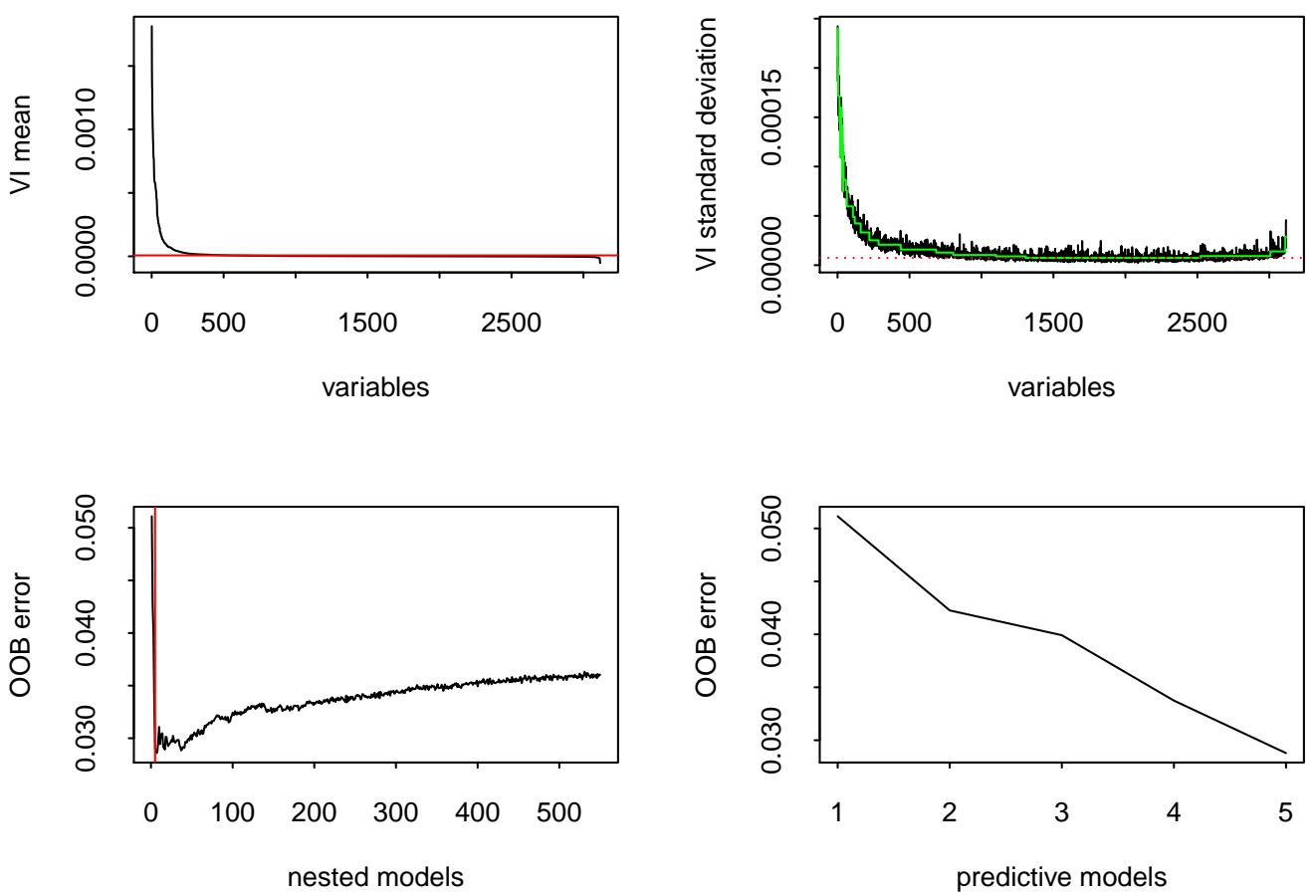

Figure 4: VSURF for the toxicity data.

We notice that after the elimination step, only 550 variables remain, thus the number of variables has been reduced by a factor of six. This ratio is not surprising since we know that there exists extreme redundancy in the gene expression data together with a lot of irrelevant variables.

$>\operatorname{plot}($ vtoxicity)

By considering the top left graph of Figure 4, it seems quite obvious to keep just a few variables. Indeed, the procedure leads to 5 variables, after both interpretation and prediction steps. Even if the numbers of selected variables are very small, they are not surprisingly low if we refer to the study in González et al. (2012), where 12 variables were selected. It would be noted that, in general, our method failed to deliver all the variables related to the response variable in the case of numerous strongly correlated predictors. In addition, we do not select the same genes but our set of selected variables and the one in González et al. (2012) exhibit strong correlations.

Even if the results are quite similar, an advantage of using VSURF is that this procedure does not involve tuning parameters unlike the procedure developed in González et al. (2012). This difference is the main reason for the gap in computation time: several minutes with 40 cores for VSURF compared to several minutes with 1 core for González et al. (2012).

\section{SRBCT data}

The dataset we consider here will allow us to apply our procedure in a classification framework. The real classification dataset is a small version of the small round blue cell tumors of childhood data and contains the expression measure of genes measured on 63 samples. This set is composed of:

- a data frame, called gene, of size $63 \times 2308$ which contains the 2308 gene expressions;

- a response factor of length 63 , called class, indicating the class of each sample (4 classes in total).

These data, presented in details in Khan et al. (2001), available in the R package mixOmics, have been widely studied but in most cases only 200 genes were considered and data have been transformed to reduce the problem to a regression problem (see e.g. Lê Cao and Chabrier, 2008). As in Díaz-Uriarte and Alvarez De Andres (2006), we consider the 2308 genes and we deal directly with the classification problem, using VSURF.

$>\operatorname{data}($ "srbct", package $=$ "mixOmics")

$>$ set.seed(10131419, "L'Ecuyer-CMRG") 


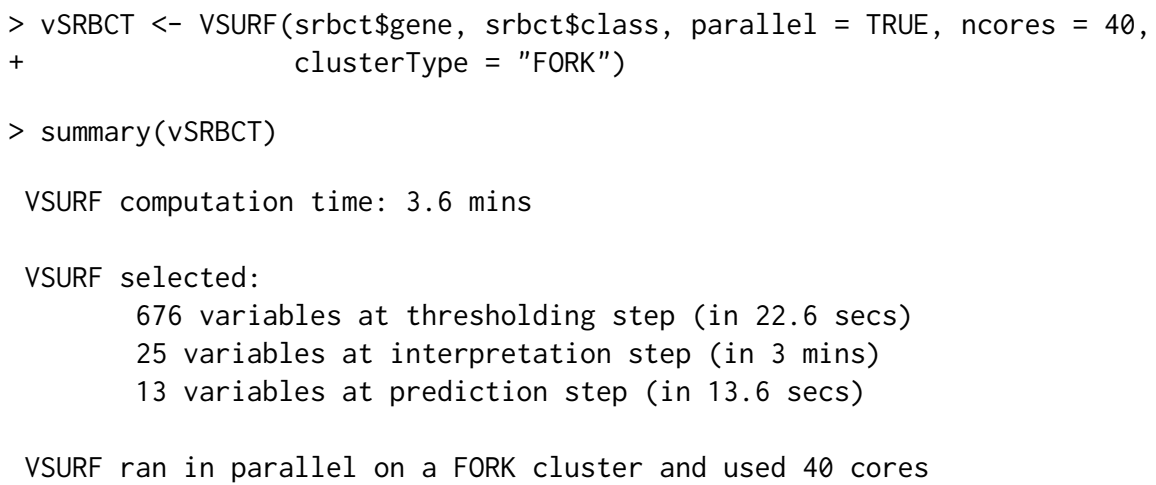

On this dataset, the procedure leads to 25 and 13 selected variables after the interpretation and prediction step respectively, and the selected variable sets are stable.

We can compare these results with those obtained in Díaz-Uriarte and Alvarez De Andres (2006) where the authors select 22 genes on the original dataset and their number of selected variables is quite stable.

To get an idea of the performance of our procedure on the dataset, we perform an error rate estimation using an external 5-fold cross-validation scheme (meaning that we apply VSURF on each fold of the cross-validation). We obtain the following error rates ${ }^{2}$ for interpretation and prediction sets respectively:

interp pred

0.015873020 .07936508

The comparison with error rates evaluated using 200 bootstrap samples in Díaz-Uriarte and Alvarez De Andres (2006) suggests that our selections are reasonable.

\section{Acknowledgements}

We thank the editor and the three anonymous referees for their thorough comments and suggestions which really helped to improve the clarity of the paper.

\section{Bibliography}

K. J. Archer and R. V. Kimes. Empirical characterization of random forest variable importance measures. Computational Statistics \& Data Analysis, 52(4):2249-2260, 2008. [p20]

R. Azen and D. V. Budescu. The dominance analysis approach for comparing predictors in multiple regression. Psychological Methods, 8(2):129-148, 2003. [p20]

A.-L. Boulesteix, S. Janitza, J. Kruppa, and I. R. König. Overview of random forest methodology and practical guidance with emphasis on computational biology and bioinformatics. Wiley Interdisciplinary Reviews: Data Mining and Knowledge Discovery, 2(6):493-507, 2012. [p19]

L. Breiman. Bagging predictors. Machine Learning, 24(2):123-140, 1996. [p20]

L. Breiman. Random forests. Machine Learning, 45(1):5-32, 2001. [p19, 20]

L. Breiman and A. Cutler. Random forest manual. 2004. URL http://www. stat.berkeley. edu/ $\sim$ breiman/RandomForests/cc_manual.htm. [p20,21]

L. Breiman, J. Friedman, R. Olshen, and C. Stone. Classification and Regression Trees. Chapman \& Hall, New York, 1984. [p19]

P. Bushel, R. Wolfinger, and G. Gibson. Simultaneous clustering of gene expression data with clinical chemistry and pathological evaluations reveals phenotypic prototypes. BMC Systems Biology, 1(1): 15, 2007. [p29]

J. M. Cadenas, M. Carmen Garrido, and R. Martínez. Feature subset selection filter-wrapper based on low quality data. Expert Systems with Applications, 40(16):6241-6252, 2013. [p20]

\footnotetext{
${ }^{2}$ The script is available at https://github.com/robingenuer/VSURF/blob/master/Example/srbct_cv.R
} 
N. Chèze, J.-M. Poggi, and B. Portier. Partial and recombined estimators for nonlinear additive models. Statistical Inference for Stochastic Processes, 6(2):155-197, 2003. [p28]

R. Díaz-Uriarte. GeneSrF and varSelRF: A web-based tool and R package for gene selection and classification using random forest. BMC Bioinformatics, 8(1):328, 2007. [p20]

R. Díaz-Uriarte and S. Alvarez De Andres. Gene selection and classification of microarray data using random forest. BMC Bioinformatics, 7(1):3, 2006. [p20, 30, 31]

R. Genuer, J.-M. Poggi, and C. Tuleau. Random forests: Some methodological insights. 2008. arXiv:0811.3619. [p26]

R. Genuer, V. Michel, E. Eger, and B. Thirion. Random forests based feature selection for decoding fMRI data. In Proceedings of COMPSTAT'2010 - 19th International Conference on Computational Statistics, pages 1071-1078, 2010a. [p21]

R. Genuer, J.-M. Poggi, and C. Tuleau-Malot. Variable selection using random forests. Pattern Recognition Letters, 31(14):2225-2236, 2010b. [p19, 20, 21, 22, 23, 27]

L. Gidskehaug, E. Anderssen, A. Flatberg, and B. K. Alsberg. A framework for significance analysis of gene expression data using dimension reduction methods. BMC Bioinformatics, 8(1):346, 2007. [p29]

I. González, K.-A. Lê Cao, M. J. Davis, and S. Déjean. Visualising associations between paired 'omics' data sets. BioData Mining, 5(1):1-23, 2012. [p29, 30]

B. Gregorutti, B. Michel, and P. Saint-Pierre. Correlation and variable importance in random forests. 2013. arXiv:1310.5726. [p20, 21]

I. Guyon and A. Elisseeff. An introduction to variable and feature selection. Journal of Machine Learning Research, 3:1157-1182, 2003. [p20]

A. Hapfelmeier and K. Ulm. A new variable selection approach using random forests. Computational Statistics E Data Analysis, 60:50-69, 2012. [p20]

T. Hastie, R. Tibshirani, and J. H. Friedman. The Elements of Statistical Learning. Springer-Verlag, New York, 2001. [p19]

A. N. Heinloth, R. D. Irwin, G. A. Boorman, P. Nettesheim, R. D. Fannin, S. O. Sieber, M. L. Snell, C. J. Tucker, L. Li, G. S. Travlos, G. Vansant, P. E. Blackshear, R. W. Tennant, M. L. Cunningham, and R. S. Paules. Gene expression profiling of rat livers reveals indicators of potential adverse effects. Toxicological Sciences, 80(1):193-202, 2004. [p29]

T. Hothorn, K. Hornik, and A. Zeileis. Unbiased recursive partitioning: A conditional inference framework. Journal of Computational and Graphical Statistics, 15(3):651-674, 2006. [p19]

J. Khan, J. S. Wei, M. Ringner, L. H. Saal, M. Ladanyi, F. Westermann, F. Berthold, M. Schwab, C. R. Antonescu, C. Peterson, and P. S. Meltzer. Classification and diagnostic prediction of cancers using gene expression profiling and artificial neural networks. Nature Medicine, 7(6):673-679, 2001. [p30]

R. Kohavi and G. H. John. Wrappers for feature subset selection. Artificial Intelligence, 97(1):273-324, 1997. [p20]

M. B. Kursa and W. R. Rudnicki. Feature selection with the Boruta package. Journal of Statistical Software, 36(11):1-13, 2010. [p20, 29]

K.-A. Lê Cao and P. Chabrier. ofw: An R package to select continuous variables for multiclass classification with a stochastic wrapper method. Journal of Statistical Software, 28(9):1-16, 2008. [p20, 30]

K.-A. Lê Cao, D. Rossouw, C. Robert-Granié, and P. Besse. A sparse PLS for variable selection when integrating omics data. Statistical Applications in Genetics and Molecular Biology, 7(1), 2008. [p29]

K.-A. Lê Cao, I. Gonzalez, and S. Dejean. mixOmics: Omics Data Integration Project, 2015. URL https://CRAN.R-project.org/package=mixOmics. R package version 5.0-4, with key contributions from F. Rohart and B. Gautier and contributions from P. Monget, J. Coquery, F. Yao and B. Liquet. [p29]

F. Leisch and E. Dimitriadou. mlbench: Machine Learning Benchmark Problems, 2010. URL https: //CRAN.R-project.org/package=mlbench. R package version 2.1-1. [p27] 
A. Liaw and M. Wiener. Classification and regression by randomForest. R News, 2(3):18-22, 2002. [p19]

G. Louppe, L. Wehenkel, A. Sutera, and P. Geurts. Understanding variable importances in forests of randomized trees. In Advances in Neural Information Processing Systems, pages 431-439, 2013. [p21]

N. Meinshausen and P. Bühlmann. Stability selection. Journal of the Royal Statistical Society B, 72(4): 417-473, 2010. [p27]

R. Nilsson, J. M. Peña, J. Björkegren, and J. Tegnér. Consistent feature selection for pattern recognition in polynomial time. Journal of Machine Learning Research, 8:589-612, 2007. [p21]

A. Peters, T. Hothorn, and B. Lausen. ipred: Improved predictors. R News, 2(2):33-36, 2002. [p20]

F. Scheipl. spikeSlabGAM: Bayesian variable selection, model choice and regularization for generalized additive mixed models in R. Journal of Statistical Software, 43(14):1-24, 2011. [p20]

C. Strobl, A.-L. Boulesteix, A. Zeileis, and T. Hothorn. Bias in random forest variable importance measures: Illustrations, sources and a solution. BMC Bioinformatics, 8(1):25, 2007. [p20]

C. Strobl, A.-L. Boulesteix, T. Kneib, T. Augustin, and A. Zeileis. Conditional variable importance for random forests. BMC Bioinformatics, 9(1):307, 2008. [p20]

V. Svetnik, A. Liaw, C. Tong, and T. Wang. Application of Breiman's random forest to modeling structure-activity relationships of pharmaceutical molecules. In Multiple Classifier Systems, pages 334-343. Springer, 2004. [p27]

T. Therneau, B. Atkinson, and B. Ripley. rpart: Recursive Partitioning and Regression Trees, 2015. URL https://CRAN. R-project. org/package=rpart. R package version 4.1-9. [p19]

A. Verikas, A. Gelzinis, and M. Bacauskiene. Mining data with random forests: A survey and results of new tests. Pattern Recognition, 44(2):330-349, 2011. [p19]

H. R. Wehrens, M. Johan, and P. Franceschi. Meta-statistics for variable selection: The R package BioMark. Journal of Statistical Software, 51(10):1-18, 2012. [p20]

J. Weston, A. Elisseeff, B. Schölkopf, and M. Tipping. Use of the zero norm with linear models and kernel methods. Journal of Machine Learning Research, 3:1439-1461, 2003. [p23]

\author{
Robin Genuer \\ University of Bordeaux \\ ISPED, Centre INSERM U-897-Epidemiologie-Biostatistique \\ 33000 Bordeaux, France \\ and \\ INRIA Bordeaux Sud Ouest, SISTM team \\ 33400 Talence, France \\ Robin.Genuer@isped.u-bordeaux2.fr \\ Jean-Michel Poggi \\ University of Orsay \\ Lab. Mathematics \\ bat 425 \\ 91405 Orsay, France \\ Jean-Michel. Poggi@math. u-psud.fr \\ Christine Tuleau-Malot \\ University of Nice Sophia Antipolis \\ CNRS, LJAD, UMR 7351 \\ 06100 Nice, France \\ Malot@unice.fr
}

